\title{
CONCEPTUAL DESIGN OF A HYPERVELOCITY ASTEROID INTERCEPT VEHICLE (HAIV) FLIGHT VALIDATION MISSION
}

\author{
Brent W. Barbee, Bong Wie ${ }^{\dagger}$ Mark Steinerł and Kenneth Getzandanner*
}

Earth has been struck in the past by near-Earth objects (NEOs) that were sufficiently energetic, in terms of mass and impact velocity, to cause significant damage ranging from local or regional devastation to mass extinctions. Such impact events will occur again in the future and humanity is beginning to see the wisdom in planning ahead to be ready to respond to the next incoming NEO so that we will have the opportunity to mount an effective defense.

Some of the key factors in designing planetary defense systems include the size of the incoming NEO and the amount of warning time. The size of the NEO determines how much damage it would cause and places limits on our response options, while the warning time further constrains our options for dealing with the NEO. Opportunities to rendezvous with NEOs at a reasonable propellant mass cost tend to occur infrequently, so for scenarios in which the NEO impact event is known less than 10 years in advance, the most viable option will likely be a hypervelocity intercept in which our mitigation system is guided to intercept the NEO at high relative velocity because the propellant cost to match the NEO's velocity would be prohibitive.

Although large NEOs are capable of causing more damage than small NEOs, the small NEOs are far more numerous and thus a small NEO impact scenario is more likely within a given time frame, all else being equal. Unfortunately, small NEOs are faint in the night sky (because they have relatively little surface area to reflect sunlight) and are therefore harder to discover and track with ground-based telescopes in advance of when they would collide with Earth. Their faintness also makes small NEOs more difficult for a spacecraft to target, especially at high relative velocity. Thus the most challenging NEO mitigation scenario involves a NEO that is small (but large enough to survive atmospheric passage and cause ground damage) and for which we have a relatively short warning time, requiring a hypervelocity intercept for deflection or destruction of the NEO. A spacecraft system capable of reliably handling that scenario would of course be able to handle less stressing cases, i.e., more warning time, lower intercept velocities, and/or larger NEOs.

Work was recently performed towards the design of such a spacecraft system by the Mission Design Laboratory (MDL) of NASA Goddard Space Flight Center's Integrated Design Center (IDC). The MDL assessed the technical feasibility of reliably performing hypervelocity interception of a $50 \mathrm{~m}$ diameter NEO and developed a conceptual design for a spacecraft and mission operations support architecture for flight validation of the system. This research was funded by and in support of the recently awarded NASA Innovative Advanced Concepts (NIAC) Phase II study entitled "An Innovative Solution to NASA's NEO Impact Threat Mitigation Grand Challenge and Flight Validation Mission Architecture Development." The goals of this research project include designing a two-body Hypervelocity Asteroid Intercept Vehicle (HAIV) that will deliver a kinetic impactor to the target NEO to excavate a shallow crater within which the second portion of the spacecraft will detonate a Nuclear Explosive Device (NED) immediately thereafter to effect a powerful subsurface detonation capable of disrupting the NEO, as shown in Figure 1. Flight validation of this system is crucial because any NEO mitigation system must be thoroughly flight tested before it can be relied upon during a true emergency. To date no such flight validations have been performed.

In this paper we present a detailed overview of the MDL study results and subsequent advances in the design of GNC algorithms for accurate terminal guidance during hypervelocity NEO intercept. The MDL study produced a conceptual configuration of the two-body HAIV and its subsystems; a mission scenario and trajectory design for a notional flight validation mission to a selected candidate target NEO; GNC results regarding the ability of the HAIV to reliably intercept small $(50 \mathrm{~m})$ NEOs at hypervelocity (typically > $10 \mathrm{~km} / \mathrm{s}$ ); candidate launch vehicle selection; a notional operations concept and cost estimate for the flight validation mission; and a list of topics to address during the remainder of our NIAC Phase II study.

\footnotetext{
*Aerospace Engineer, NASA/GSFC, Code 595, 8800 Greenbelt Road, Greenbelt, MD 20771, USA.

†Vance Coffman Endowed Chair Professor, Asteroid Deflection Research Center, Department of Aerospace Engineering, Iowa State University, 2271 Howe Hall, Room 2325, Ames, IA 50011-2271, USA.

$\ddagger^{\ddagger}$ Mission Design Lab Team Lead, NASA/GSFC, Code 592, 8800 Greenbelt Road, Greenbelt, MD, 20771, USA.
} 

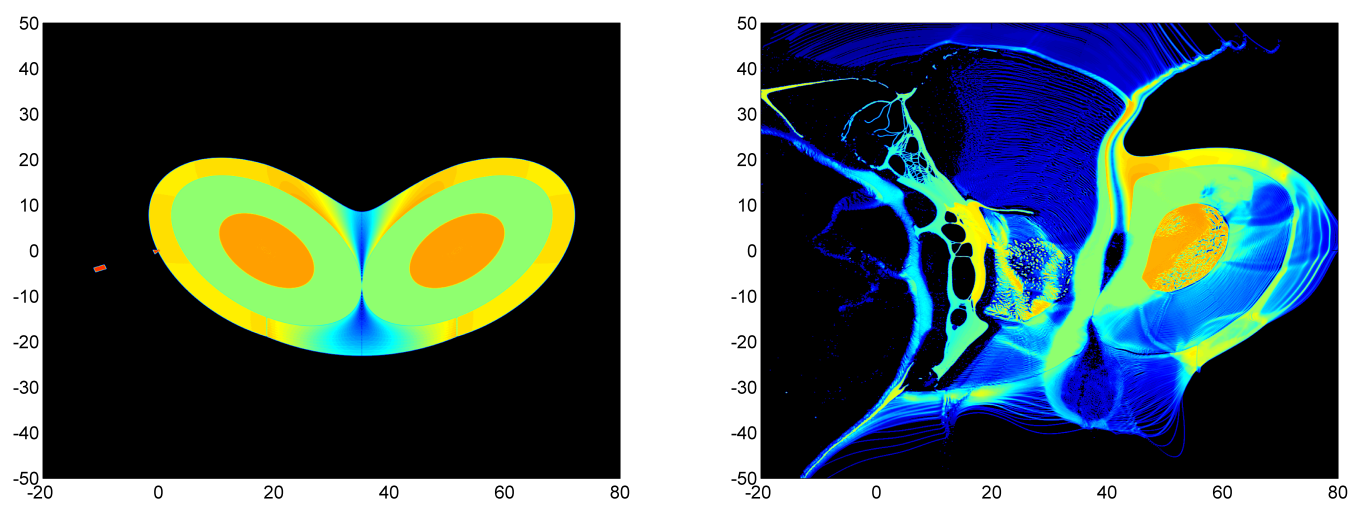

Figure 1. Simulated disruption of a small asymmetrically shaped NEO by a subsurface NED detonation. A NED detonated just below the surface of a NEO is approximately 20 times more effective at disrupting the NEO than if the NED were merely placed on the NEO's surface.

The HAIV system and mission architecture were initially developed during a NIAC Phase I research study. The Phase II research is focused on further design of the system and development of a flight validation mission concept to demonstrate the capability to handle short warning time $(<10$ years $)$ NEO impact scenarios involving the more numerous smaller NEOs in the 50-100 m size category. Phase II tasks include three-dimensional hypervelocity impact and nuclear explosion modeling and simulation using next-generation GPU computers; HAIV design trades, including concepts for leader/follower vehicle separation such as a 10$20 \mathrm{~m}$ deployable boom versus free-flying (i.e., not physically connected) vehicles; study of various options for hypervelocity impact detectors/sensors; and design of GNC algorithms to enable robust and reliable precision impact with a small (50-100 m) irregularly shaped rotating NEO using advanced optical navigation and guidance logic. Ultimately our Phase II research will lead to the development of a proposal for a flight validation mission of the HAIV system, ideally within a mission cost cap of approximately $\$ 500 \mathrm{M}$. We are also exploring the possible benefits of conducting ground experiments using scaled HAIV models instrumented with candidate hypervelocity impact sensors/detectors.

One of the primary objectives of the MDL study was assessment of the feasibility of accurately intercepting a small NEO with a spacecraft at high relative velocity. We chose to examine mission scenarios involving a NEO between 50 and $100 \mathrm{~m}$ in diameter and a velocity relative to the NEO at intercept of approximately $11.5 \mathrm{~km} / \mathrm{s}$. We sought to assess the capability of the system design to achieve impact within $10 \mathrm{~m}$ of the center of the NEO with $3 \sigma$ confidence. To accomplish this, the MDL designed and analyzed a two-body HAIV, along with supporting mission and ground architecture, capable of reliably delivering a notional NED payload to a target NEO and returning adequate telemetry for validation of the system performance.

The trade space for selecting some of the desired flight validation mission target NEO characteristics is shown in Figure 2(a). For the notional flight demo target we selected from among known NEOs whose orbits are completely interior or exterior to the Earth's orbit to ensure that the flight validation mission would have no chance of causing the NEO, or fragments of it, if any, to subsequently impact the Earth. We performed intercept trajectory scans to identify candidate targets based on their orbital and physical characteristics in the context of our mission design criteria. The notional target we selected is near-Earth asteroid (NEA) $2006 \mathrm{CL}_{9}$. This NEA has an absolute magnitude (a measure of optical brightness) of 22.73 , which corresponds to a diameter of $104 \mathrm{~m}$ if its geometric albedo is $13 \%$ or $75 \mathrm{~m}$ if its geometric albedo is $25 \%$ This NEA has a measured rotation period (derived from photometry in the form of lightcurve data) of 0.145 hours $\pm 30 \% 2^{2}$. According to NASA's NHATS websit $3^{3}$, this NEA may be observable again in June of 2014. Such future observations may provide an opportunity to improve its Orbit Condition Code (OCC),

\footnotetext{
${ }^{1}$ The albedo of a particular NEO is generally not well known, so we use representative values. For more information see: http://neo.jpl.nasa.gov/neo/groups.html, http://neo.jpl.nasa.gov/glossary/albedo.html and http://www. minorplanetcenter.net/iau/lists/Sizes.html

2 http://ssd.jpl.nasa.gov/sbdb.cgi?sstr=2006\%20CL9

3 http://neo.jpl.nasa.gov/nhats/

4 http://neo.jpl.nasa.gov/cgi-bin/nhats?sstr=2006\%20CL9\&dv=12\&dur=450\&stay=8\&launch=2015-2040
} 


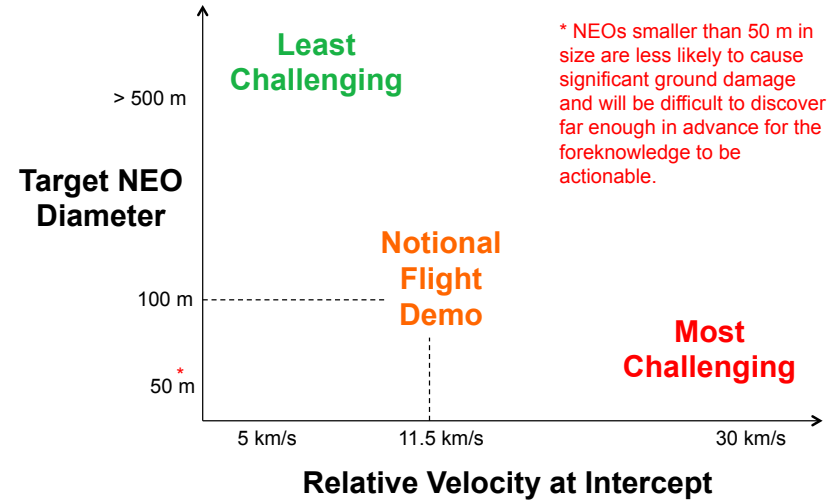

(a) Depiction of the trade space for selecting a target NEO for a flight demo mission.

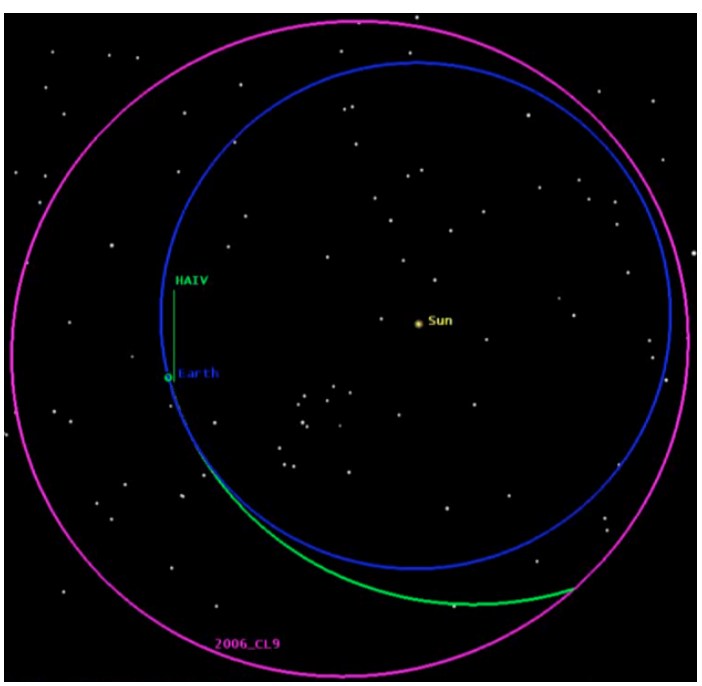

(b) Ecliptic plane projection of the orbits of Earth (blue) and notional flight demo target NEA 2006 CL9 (violet), along with the HAIV intercept trajectory (green).

Figure 2. Trade space for selecting a target NEO for a flight demonstration mission and the mission trajectory for the selected notional target, $2006 \mathrm{CL}_{9}$.

a measure of along-track orbit knowledge uncertainty also known as the MPC Uncertainty Parameter 5 from the current value of 5 (where 0 represents a well known orbit and 9 represents a very poorly known orbit). In terms of orbit properties, this NEA belongs to the Amor group (Mars crossing, Earth approaching), with a semi-major axis of $1.35 \mathrm{AU}$, an eccentricity of 0.237 , and an inclination of $2.9^{\circ}$.

The notional mission trajectory, shown in Figure 2(b), departs Earth on August 2, 2019 with an Earth departure $C_{3}$ energy of $11.99 \mathrm{~km}^{2} / \mathrm{s}^{2}$ and intercepts the NEA 121.41 days later with a relative velocity at intercept of $11.5 \mathrm{~km} / \mathrm{s}$ and an approach phase angle of $3.04^{\circ}$. The small approach phase angle places the Sun nearly behind the spacecraft during terminal approach, providing excellent natural illumination of the target. The trajectory design for the spacecraft requires only $58 \mathrm{~m} / \mathrm{s}$ of $\Delta V$ for deterministic and statistical mid-course correction maneuvers, calibration burns, ACS maneuvers, and terminal guidance phase maneuvers, which include maneuver execution errors and relative navigation uncertainties. The spacecraft's maximum distances from the Earth and Sun are 0.36 AU and 1.28 AU, respectively. The spacecraft's $\Delta V$ budget requires only $64 \mathrm{~kg}$ of hydrazine propellant and pressurant, while the spacecraft carries $363.8 \mathrm{~kg}$; this provides substantial $\Delta V$ margin. The wet mass at launch for the spacecraft is $1501 \mathrm{~kg}$, which is $814 \mathrm{~kg}$ less than the $2315 \mathrm{~kg}$ capability of the Atlas V 401 launch vehicle to the aforementioned Earth departure $C_{3}$ energy of $11.99 \mathrm{~km}^{2} / \mathrm{s}^{2}$. Thus we have $54.3 \%$ mass margin.

The particulars of the NED payload (design, selection, fuzing, etc.) were purposely placed outside the scope of the MDL study. This is consistent with the plan for the flight validation mission, which would only carry a simple dummy mass as a surrogate for the NED payload. In the MDL study we modeled this notional dummy NED payload as a $300 \mathrm{~kg}$ cylinder $1 \mathrm{~m}$ in length with a $0.5 \mathrm{~m}$ face diameter. The spacecraft design includes thermal and debris protection for the NED in the form of a conical structure having a Kevlar skin and aluminum alloy core honeycomb construction with either thermal coatings applied or high temperature foam injected into the cavity surrounding the NED payload. The conceptual design of the HAIV produced by the MDL is shown in Figure 3

The terminal guidance analysis is one of the most challenging aspects because the high relative velocity produces a short final approach time line, with automated guidance beginning 2 hours prior to impact. We modeled a notional optical navigation camera using parameters from the Deep Impact mission's Impact Targeting Sensor (ITS), including a Field of View (FOV) of $0.6^{\circ}$, a focal length of $2101 \mathrm{~mm}$, and $1024 \times$ 1024 resolution. Our requirement for optical acquisition of the target NEO is that it must have an apparent

$\sqrt[5]{\text { http: //www.minorplanetcenter.net/iau/info/UValue.html }}$ 


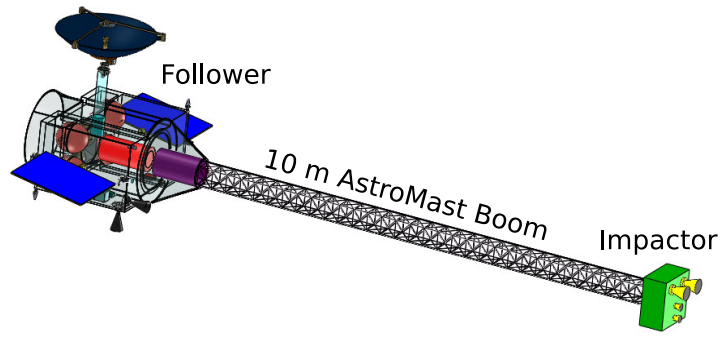

(a) Conceptual HAIV design showing the follower, boom, and impactor vehicle components.

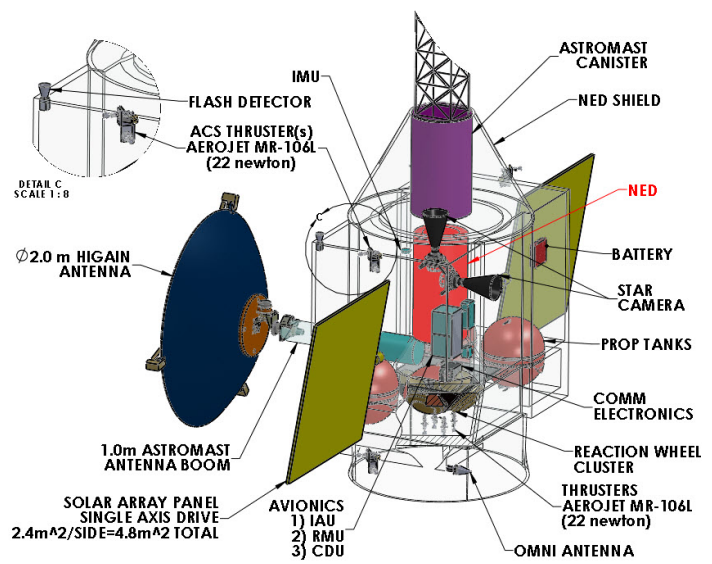

(b) Detail view of the follower portion of the two-body HAIV showing selected subsystem components.

Figure 3. Conceptual design for the two-body HAIV, including $10 \mathrm{~m}$ boom, produced by the MDL study.

magnitude of 13 with a signal-to-noise ratio (SNR) of 10 within a 5 second exposure. A sequential Kalman filter was used for the relative state estimation during terminal guidance and multiple measurement error sources were modeled, including spacecraft state knowledge errors, attitude knowledge errors, centroid pixel noise, and maneuver execution errors.

A Monte Carlo analysis was performed for the terminal guidance phase showed that $56 \%$ of the cases meet the $10 \mathrm{~m}$ accuracy requirement, yielding a $3 \sigma$ accuracy of $24 \mathrm{~m}$ around the NEO center. These results are visualized in Figure 4. Thus, one of the outputs of the MDL study was a list of additional research tasks to improve terminal guidance to achieve $10 \mathrm{~m}(3 \sigma)$ impact accuracy.

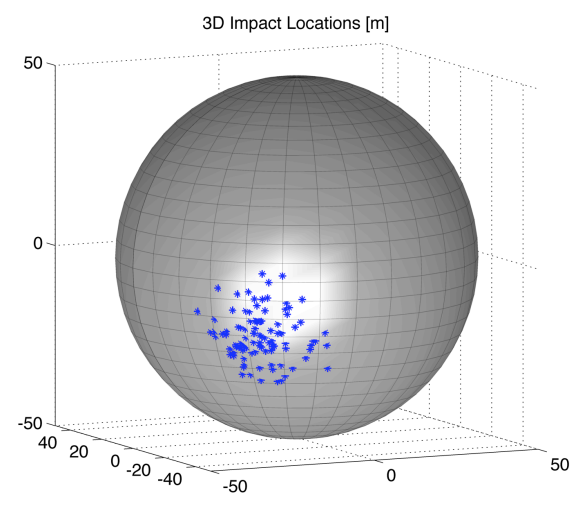

Figure 4. Simulated HAIV impact locations on the notional target NEO from Monte Carlo simulation. The area of brightness on the three-dimensional spherical NEO model accurately depicts the direction of solar illumination in our mission scenario.

In this paper we will provide further details regarding the MDL results for the HAIV spacecraft and mission architecture design in the context of a future flight validation mission. We will also present new results obtained subsequent to the MDL study in which we have made progress in both the research areas originally outlined in our Phase II research plan and those identified by the MDL study. In particular, we will focus on new results in the development of advanced GNC algorithms for precision hypervelocity impact with small (50 m diameter) NEOs across a range of representative simulation scenarios. These new results build upon the preliminary results shown herein from the MDL study. 